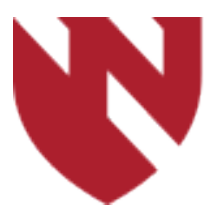

December 2019

\title{
Falsely Elevated Thyroglobulin in a Patient with Thyroid Cancer and Rheumatoid Arthritis after Adalimumab Therapy
}

\author{
Archana Purushothaman \\ University of Nebraska Medical Center \\ Nicholas Lintel \\ University of Nebraska Medical Center \\ Kaitlin Brau \\ University of Nebraska Medical Center \\ Whitney Goldner \\ University of Nebraska Medical Center
}

Tell us how you used this information in this short survey.

Follow this and additional works at: https://digitalcommons.unmc.edu/gmerj

Part of the Higher Education Commons, and the Medicine and Health Sciences Commons

\section{Recommended Citation}

Purushothaman, A., Lintel, N., Brau, K., , Goldner, W. Falsely Elevated Thyroglobulin in a Patient with Thyroid Cancer and Rheumatoid Arthritis after Adalimumab Therapy. Graduate Medical Education Research Journal. 2019 Dec 13; 1(1).

https://digitalcommons.unmc.edu/gmerj/vol1/iss1/65

This Conference Proceeding is brought to you for free and open access by DigitalCommons@UNMC. It has been accepted for inclusion in Graduate Medical Education Research Journal by an authorized editor of DigitalCommons@UNMC.For more information, please contact digitalcommons@unmc.edu. 


\section{Falsely Elevated Thyroglobulin in a Patient with Thyroid Cancer and Rheumatoid Arthritis after Adalimumab Therapy}

\section{Creative Commons License}

\section{c) (1) $\Theta($}

This work is licensed under a Creative Commons Attribution-Noncommercial-No Derivative Works 4.0 License. 


\section{Falsely Elevated Thyroglobulin in a Patient with Thyroid Cancer and Rheumatoid Arthritis after Adalimumab Therapy \\ Archana Purushothaman, Nicholas Lintel, Kaitlin Brau, Whitney Goldner}

\author{
Mentor: Whitney Goldner \\ Program: Diabetes, Endocrinology, and \\ Metabolism
}

Introduction: Thyroglobulin (TG) elevation is used as a tumor marker for evidence of recurrence or metastatic disease of thyroid carcinoma.TG assays are usually immunoassays which are subject to a number of interferences.

Case: We report a middle-aged patient with rheumatoid arthritis (RA) and Follicular Variant of Papillary Thyroid Carcinoma, AJCC 7, stage T2N0M0, status post total thyroidectomy and RAI ablation, with ATA excellent response to therapy. Ten years after diagnosis, the patient presented with sudden elevation in TG of $111 \mathrm{ng} / \mathrm{mL}$. On repeat testing, TG ranged from 111-300 ng/mL with undetectable TG ab. Imaging did not reveal evidence of recurrent disease. On further investigation, the patient reported starting adalimumab for rheumatoid arthritis one-year prior. Tenfold dilution of the sample showed a decrease in TG from 300 to $47 \mathrm{ng} / \mathrm{mL}$ in a non-linear manner, suggestive of interference. The serum was treated with blocking agents and the TG decreased to $2.7 \mathrm{ng} / \mathrm{mL}$ suggesting the presence of HAMA. TG testing via mass spectrometry was undetectable.

Conclusion: Heterophilic abs like

Rheumatoid Factor (RF) or HAMA are human abs that interact with assay abs. In 2-site immunometric "sandwich complex" assays, they often generate false-positive results. Those with RA are at risk of interference from $\mathrm{RF}$ or they can generate abs from exposure to immunotherapies that use chimeric abs. Adalimumab is a fully human monoclonal ab, so not expected to result in production of HAMA. The assay interference in this case is likely due to either RF or HAMA from an unknown source.

https://doi.org/10.32873/unmc.dc.gmerj.1.1.065

\section{A Rare Case of Lung Cancer Metastasis to Thyroid} Thiyagarajan Thangavelu, Whitney S Goldner

\section{Mentor: Whitney S Goldner}

Program: Diabetes, Endocrinology, and Metabolism

Background: Most common tumors in thyroid are well differentiated thyroid malignancies (Papillary and Follicular). Metastasis to thyroid gland are rare despite thyroid being a very vascular organ. Lung cancer commonly metastasize to liver, adrenals, brain and bones. Here we present a rare case of non-small cell lung cancer metastasis to a previously benign thyroid nodule.

Methods: An elderly patient was followed at the Endocrine clinic for multinodular goiter for several years. The patient had biopsy of these nodules multiple times in the past and have been benign. In 2016, the patient was diagnosed with large cell lung cancer and was treated with resection and chemotherapy.

In 2017, an ultrasound showed increase in size of the right thyroid nodule. The patient underwent FNA and it showed indeterminate results with rare atypical cells suspicious of carcinoma. It was sent for molecular testing and it was negative for thyroid malignancy.

Results: The slides were reviewed by the pathologist again and compared to the prior lung tumor specimens and it showed similar features to the lung carcinoma. CT scan showed prominent thyroid mass with enlarged paratracheal lymph node. The patient underwent total thyroidectomy and lymph node removal for definitive diagnosis. Pathology confirmed non-small cell lung carcinoma.
Conclusions: In a patient with another known primary cancer, metastasis to thyroid should be considered in the differential of thyroid nodule. Imaging alone will not differentiate between primary and secondary thyroid malignancies. Molecular markers for thyroid cancer if negative do not rule out metastasis from another primary malignancy. FNAC with histochemistry and comparing to primary tumor help make the diagnosis.

https://doi.org/10.32873/unmc.dc.gmerj.1.1.066

\section{Cachexia Index: A Marker for Survival in Pancreatic Cancer?}

Mridula Krishnan, Lei Yu, Osayande Ferdinand O, Aneesha Dasgupta, Lyudmyla Berim, Jennifer Oliveto, Pankaj Singh, Fang Yu, Alam Morshed

Mentors: Lyudmyla Berim and Jennifer Oliveto

Program: Radiology

Introduction: The incidence of pancreatic cancer is on the rise and continues to have a poor overall survival despite advancement in treatment strategies. The effect of sarcopenia on the prognosis of patients with pancreatic cancer is unknown.

Methods: We performed a single-center retrospective study at our institution by measuring muscle mass among patients with pancreatic cancer from CT scan at initial diagnosis and at 8 weeks post-treatment. The results were stratified based on the percentage of muscle loss $(<10 \%$ and $>10 \%)$ to determine whether sarcopenia impacted outcomes primarily, overall survival (OS) and progression-free survival (PFS).

Results: This retrospective study included 162 patients from the University of Nebraska medical center diagnosed between 2009 and 2017 with stage I-IV pancreatic cancer. We calculated hazard ratios (HRs) to evaluate the associations of overall survival with sarcopenia. There is a significant association between improved OS and PFS among those with less intensive reduction in muscle mass ( $p=0.038, p=0.027$ respectively). However, no significant association of BMI loss with overall mortality on either OS or PFS ( $\mathrm{p}=0.80$, $\mathrm{p}=0.65)$.

Conclusion: The presence of sarcopenia may be a predictor of prognosis among patients with pancreatic cancer. A better understanding of the molecular mechanisms of sarcopenia might help improve survival outcomes in pancreatic cancer.

https://doi.org/10.32873/unmc.dc.gmerj.1.1.067 